Elsevier required licence: (C) <2021>. This manuscript version is made available under the CC-BY-NCND 4.0 license http://creativecommons.org/licenses/by-nc-nd/4.0/

The definitive publisher version is available online at

[https://www.sciencedirect.com/science/article/pii/S016885102100049X?via\%3Dihub] 


\title{
Physicians' View and Experiences of Defensive Medicine: An International Review of Empirical Research
}

\begin{abstract}
This study systematically maps empirical research on physicians' views and experiences of hedging-type defensive medicine, which involves providing services (eg, tests, referrals) to reduce perceived legal risks. Such practices drive over-treatment and low value healthcare. Data sources were empirical, English-language publications in health, legal and multidisciplinary databases. The extraction framework covered: where and when the research was conducted; what methods of data collection were used; who the study participants were; and what were the study aims, main findings in relation to hedging-type defensive practices, and proposed solutions.
\end{abstract}

79 papers met inclusion criteria. Defensive medicine has mainly been studied in the United States and European countries using quantitative surveys. Surgery and obstetrics have been key fields of investigation. Hedging-type practices were commonly reported, including: ordering unnecessary tests, treatments and referrals; suggesting invasive procedures against professional judgment; ordering hospitalisation or delaying discharge; and excessive documentation in medical records. Defensive practice was often framed around the threat of negligence lawsuits, but studies recognised other legal risks, including patient complaints and regulatory investigations. Potential solutions to defensive medicine were identified at macro (law, policy), meso (organisation, profession) and micro (physician) levels.

Areas for future research include qualitative studies to investigate the behavioural drivers of defensive medicine and intervention research to determine policies and practices that work to support clinicians in de-implementing defensive, low-value care.

Keywords: defensive medicine; low value care; clinical decision-making; legal aspects; review

Highlights 3 to 5 bullet points (maximum 12 words per bullet point)

- Physicians across different healthcare and legal systems commonly report hedging-type defensive practice. 
- Most research is quantitative and focused on certain areas like surgery and obstetrics.

- Qualitative research should investigate the factors that amplify or alleviate legal fears.

- Intervention points exist at macro, meso and micro levels to reduce defensive practice. 


\section{INTRODUCTION}

Medical overuse and low value care are matters of serious concern in contemporary health policy and practice. ${ }^{1}$ These terms refer to care that has no benefit or where the harms or costs of care disproportionately exceed potential benefits. ${ }^{2} \mathrm{~A}$ growing body of research depicts a complex set of drivers and possible solutions to the problem of low value care., Examples of drivers include medicalisation and the belief that more is better, financial incentives, new technologies, and a lack of knowledge and confidence among professionals and patients about how to avoid overuse. ${ }^{3}$

Fear of legal liability is often cited as a driver of low value care, when clinicians order tests and procedures, make referrals, and prescribe drugs to reduce perceived legal risks, rather than to advance patient care. By definition, defensive practice has little clinical value, but clinicians do it 'just in case' the tests or treatments might allay future litigation or complaints. In addition to these hedging-type behaviours, defensive practices may also involve avoidance behaviours, which occur when clinicians avoid particular practice areas or patients perceived as high risk. Hedging behaviours are the focus of this article. The terms 'assurance' behaviour and 'positive' defensive practice are also used in the literature, however, as these descriptors may imply beneficial actions and outcomes we prefer the term 'hedging' behaviour, as proposed by Bourne et al. ${ }^{5}$

Much has been written about defensive medical practice. The influence of tort law on defensive behaviour has been a key area of investigation, however several analyses conclude that changing the policy settings through macro-level law reform may be insufficient to discourage defensive behaviour and improve care quality. ${ }^{6,7}$ Understanding the behavioural drivers of defensive practice is a key area for further research; it is argued that "overuse drivers go beyond incentives and culture and that reducing overuse will require deeper understanding of physician behaviour." ${ }^{1(\text { p26) }}$ A clear picture of the state of empirical research on defensive practice is valuable to understand the current knowledge base, to inform further research, and to design evidence-informed interventions that support clinicians in de-implementing defensive practices that contribute to medical overuse. $^{8}$ 
This study aimed to systematically map the literature on research that collected empirical data to investigate physicians' views and experiences in relation to hedging-type defensive medical practice. This approach to a structured literature review examines the features of available knowledge before determining how it can be used further; ${ }^{9}$ the purpose is not to appraise the rigour of the evidence in relation to a specific question or to provide a quantitative estimate of the relationship between factors involved. Our review reports on the key features of this body of research, focused on the ' $W$ ' questions: where and when the research was conducted; what methods of data collection were used; who the study participants were; and what were the study aims, main findings in relation to hedging-type defensive practices, and proposed solutions to this behaviour.

\section{METHOD}

Papers were eligible for inclusion in our review if they were published in English and reported on studies involving primary data collection that investigated hedging-type defensive practices among physicians. This latter criterion captured papers that reported on the performance of actions or provision of services to reduce perceived legal risks, such as ordering tests and procedures or making specialist referrals. Papers were not eligible if they focused on avoidance-type behaviours, such as doctors leaving the practice of obstetrics due to legal risks. A search of academic databases SCOPUS, Academic Search Complete (EBSCO), HeinOnline and MEDLINE with no date restrictions was conducted in September 2018, with an updated search in October 2019. As defensive medicine is a medical, legal and social phenomenon, these databases were selected to ensure wide cross-disciplinary coverage. Searches used the standardized Medical Subject Heading (MeSH) term 'defensive medicine', as well as 'defensive med' or 'defensive practice' as keywords. Search results were loaded to the EPPI-Reviewer software, Version 4.11.0.0.

Titles and abstracts ( $n=2045$, with duplicates removed) were screened by a trained research assistant to exclude search results using the following criteria: not in English; inappropriate article type (studies not involving primary data collection, commentary-style articles, focus on avoidance-type practice); or article clearly irrelevant (eg, defensive practice used in a different context; for example, the search returned articles on concussion litigation for athletes who played defensive positions and defensive traits in psychiatric personality disorders). Search results that returned only a title without a locatable abstract were also 
excluded if the title indicated the article was likely irrelevant (eg, title referred to an inappropriate article type, such as a commentary piece, or an irrelevant context such as sports). Titles that suggested potential relevance were included for full-text screening. Of 156 papers screened on full-text, the following exclusion criteria were applied: hedging-type defensive practice mentioned but not the focus; or inappropriate article type (eg, a commentary on an empirical study; secondary analysis of data, such as Medicare claims). Through this process, 65 papers were included for data extraction. Reference lists for the most recent decade (2009-2019) of included papers were checked to identify any further relevant papers $(n=14)$. In total, 79 papers were included in our review. See Figure 1 for the literature search flow diagram.

Figure 1: Literature search flow diagram [Figures and Tables appear at the end of this manuscript]

The extraction framework was developed through discussion between the lead author and a research assistant and data extraction criteria were piloted on five articles. The extraction framework covered the following descriptive data: country where research was undertaken; year of publication; data collection method (eg, survey, focus group); participant group by area of medical practice/specialty; medico-legal risk considered in the study (eg, malpractice litigation, complaints, disciplinary proceedings). Also extracted and reported here in narrative format were the aims of the paper, results related to hedging-type behaviours and potential solutions to reduce defensive practice. In extracting solutions, we looked first for statements that explicitly proposed strategies. In the absence of explicit statements, we inferred solutions as interventions to address problems identified by the research. Data were extracted by the lead author (initials removed) and a research assistant with a second investigator (initials removed) providing review to ensure consistency and accuracy. Any discrepancies were resolved through discussion and consensus.

\section{RESULTS}

The Table of Included Studies summarises the extracted data for all 79 papers. It is provided as a separate file due to its length.

\section{Country of study}


Figure 2 reports the number of publications by country in which the research was undertaken. Nearly all research has been conducted in a single country, with four papers reporting on data collected in more than one country to generate comparative data on defensive practice. ${ }^{10-13}$ The bulk of research on defensive practice has been conducted in the United States and European countries. Single-country papers reporting on American data $(n=29)$ comprised $36.7 \%$ of the literature included in this review, followed by studies from the UK ( $n=9,11.4 \%)$, with one major study by Bourne et al accounting for three of these UK publications. ${ }^{5,14-21}$ All of the multi-country studies involved data collection in the US, increasing the proportion of papers with American data to $41.8 \%$ of the included studies. One international survey study collected data from respondents in 74 countries. ${ }^{10}$ [insert Figure 2 here]

\section{Year of publication}

Figure 3 shows the frequency count of publications across five-year time intervals, revealing a steady upward trend in the number of articles and a large increase from 2011 onwards. Two-thirds of the 79 articles were published since $2011(n=53 ; 67 \%)$.

[insert Figure 3 here]

\section{Study design / data collection method}

Cross-sectional surveys were the most common method of data collection ( $n=64 ; 81 \%)$, mostly producing quantitative data. Surveys varied in their format and length, with some framing questions around clinical scenarios ${ }^{\text {see eg } 22-25}$ and others asking respondents to reflect on their most recent defensive behaviours. ${ }^{\text {see eg } 17,26,27}$ One paper reported a longitudinal study of emergency medicine residents that measured the baseline and evolution of malpractice concerns and defensive practice from the start to finish of a fouryear residency program. ${ }^{23}$

Just five publications (6.3\% of the papers reviewed) reported qualitative data collected via interviews or focus groups with clinicians ${ }^{21,28-31}$. Of these, two involved interviews as a follow-up to a survey. ${ }^{21,31}$ Several publications provided a qualitative analysis of open text responses collected as part of a survey. ${ }^{14,32,33}$ Only one study was a quasi-experimental educational intervention with a module on the drivers of low value care, including fear of 
legal risks. ${ }^{34}$ Pre- and post-intervention surveys collected data on trainees' attitudes toward defensive practice and their tolerance of uncertainty. Performance on high value care questions in a national medical exam was also measured.

Several papers reported on prospective audit studies involving data collection at the time of clinical decision-making. For example, in hospital clinic, emergency and trauma settings, doctors were asked to rate the defensive motivations (if any) of decisions, such as all tests ordered by hospitalists in one day, ${ }^{35}$ imaging orders by orthopaedists, ${ }^{36}$ orders for CT scans ${ }^{37}$ or CT angiography, ${ }^{38}$ or management of patients with possible acute coronary syndrome. ${ }^{39}$ In the primary care context, two papers reported on defensive practices in tests, procedures and referrals: one was a US study of fixed-salary physicians ${ }^{40}$ and the other was a Dutch study in which family doctors rated the defensiveness of laboratory testing and diagnostic imaging over a one year period. ${ }^{41}$

\section{Participant group by area of medical practice}

The studies examined defensive practice in various fields of medicine. Table 1 reports the fields of practice investigated in five or more papers.

[insert Table 1 here]

Just over a quarter of the papers $(26.6 \%, n=21)$ reported on studies that involved a mixed sample of clinician participants. These included studies where surveys were distributed through national medical associations or multiple hospitals/departments and doctors from various specialities responded. Of the mixed sample papers, four reported on studies that involved medical and other healthcare professionals, such as nurses and midwives. ${ }^{21,22,29,42}$ One of these studies also involved lawyers and healthcare consumers with an interest in perinatal litigation. ${ }^{21}$

The next largest proportion of papers reported on defensive practice in surgery: 16 papers (20.3\%) reported exclusively on surgeons; a further 15 of the mixed sample studies included general or specialist surgeons. Neurosurgery was the most investigated surgical specialty field: nine papers investigated only neurosurgeons ${ }^{11,11,43-49}$ and three of the mixed sample papers included neurosurgeons. ${ }^{25,27,50}$ Eleven papers reported on general surgery or surgery without identifying a specialty area. Trauma and/or orthopaedic surgery were covered in 
four papers, ${ }^{37,51-53}$ (one also involved radiologists ${ }^{51}$ ) and two of the mixed sample papers included this surgical specialty. ${ }^{27,50}$ Nine papers focused on general/family practitioners and a further four included this group in a mixed sample study; overall $16.5 \%$ of the included papers reported the views of this practitioner group.

Areas of medical specialty investigated in under five publications included:

cardiology, ${ }^{12,24,25,50}$ oncology, ${ }^{54,55}$ pathology, ${ }^{56,57}$ gastroenterology, ${ }^{58,59}$ paediatrics, ${ }^{50,60}$ infectious diseases/clinical microbiology ${ }^{10}$ and otolaryngology. ${ }^{61}$

Nine papers reported on studies that involved medical students or those in training programs (eg, residencies, fellowships); students and trainees were exclusively sampled in five studies ${ }^{13,23,32,34,62}$ and were included as part of a mixed sample in four studies. ${ }^{12,60,63,64}$

A few studies purposively sampled practitioners who had experience of lawsuits or complaints (eg, Nakamura et al's Japanese study of surgeons who had been sued ${ }^{65}$; Bourne et al's UK research on doctors who had experienced complaints $\left.{ }^{5}\right)$. More commonly studies asked about respondents' legal history as a demographic question (eg, prior history of being the subject of a lawsuit or complaint and/or experience of seeing colleagues involved in legal processes).

\section{Nature of medico-legal risk considered as a driver of defensive practice}

Just over half of papers $(54.5 \%, n=43)$ focused on malpractice/negligence litigation as the medico-legal risk driving defensive behaviours. Nearly $40 \%$ of papers $(37.8 \%, n=30)$ considered various medico-legal claims and processes that clinicians could experience. In this category of papers, 11 (14\% of total) focused on malpractice litigation and complaints. The other 19 papers ( $24 \%$ of total) considered a broader range of risks, including malpractice litigation, complaints, internal investigations by management, professional disciplinary proceedings, criminal prosecutions and coronial investigations. A few of these papers also considered the risk of verbal or physical assault by patients and negative publicity. Of all the papers, six considered complaints exclusively (7.6\%); three of these papers were from one large study by Bourne et al that sampled UK doctors who had experience of being the subject of a complaint.

\section{Aims of the included papers}


The primary aims stated in most publications related to investigating practitioners' beliefs, attitudes, practices and experiences in relation to defensive practice. For example, a common aim was to quantify the types and prevalence of defensive practices, as well as to investigate perspectives such as the extent to which practitioners perceived their patients as potential litigants/complainants, the existence of a 'malpractice crisis', and their future risk of being named in a lawsuit or complaint. The impact of attitudes and experiences on the propensity to engage in defensive practice was also commonly investigated. Several studies had an additional aim of quantifying costs associated with defensive practice. see eg $37,40,52,66$ Uniquely, one study investigating defensive CT scans for trauma patients sought to quantify the impacts for patients in terms of unnecessary radiation exposure. ${ }^{37}$

\section{Main findings}

Fear of legal risks and defensive practices are frequently reported by practitioners in different countries and working in a variety of medico-legal systems. Prevalence of hedgingtype defensive practices was commonly reported by over $70 \%$ of respondents. Many types of defensive practices were reported, including: ordering more tests, treatments and procedures than medically indicated; unnecessary referrals to specialists; suggesting invasive procedures to patients against professional judgement; prescribing more medication than necessary; ordering hospitalisation or delaying discharge for patients who could be managed through outpatient care; and inserting in medical records superfluous or excessive information or remarks that would not have been included were practitioners not concerned about legal risks.

Papers that distinguished the degree of defensiveness of practices revealed that highly defensive practices (ie, mostly or completely provided due to legal worry) were less common $^{\text {see eg } 25,35,40,41}$, but many practices were at least partially motivated by a desire to avoid legal risks. When asked, many respondents, especially surgeons, reported that they viewed patients as potential legal threats. ${ }^{11,44,46-48,50,67}$ Experiencing lawsuits or complaints was associated in some studies with more subsequent defensive practice. ${ }^{\text {see eg } 6,43,47,62,72-74}$ Some studies also indicated that younger/less experienced practitioners ${ }^{\text {see eg } 16,17,46,64,68}$ and those who frequently worried about legal risks also engage in more defensive practice. ${ }^{\text {see eg }}$ 
The several studies that involved data collection in more than one country reported a few significant differences. A study of neurosurgeons in the US, South Africa and Canada found that a majority of respondents in all three countries reported engaging in defensive practice, but this behaviour was reported by $65 \%$ of Canadian respondents compared to $85 \%$ of American and South African respondents (Yan 2016). The latter were more likely to perceive a "malpractice crisis". The authors suggested features of the healthcare system and medicolegal context that could influence these differences, such as lower malpractice premiums in Canada and medical defence provided by a single, national organisation. Neurology residents in the US were more likely than their German counterparts to agree that litigation as an "important problem," however residents in both countries reported similar experiences with supervising physicians teaching them about litigation risks (Brilla, 2006). Cardiologists in the US and China reported similar worries about malpractice risks; the only significant difference was that US physicians were more sceptical that following national guidelines offered protection from liability (Badri, 2014). A multi-country survey of infectious disease specialists and clinical microbiologists reported some differences, such as practitioners in the UK and Italy being more fearful of legal risks than those in France and Germany (Tebano, 2018); as an exploratory study, the authors recommended further comparative research.

\section{Potential solutions}

The papers indicated various solutions to reduce defensive practice, which suggest potential opportunities for interventions at macro (system), meso (organisation) and micro (practitioner) levels (see Figure 4). At the macro level, reforms to liability and compensation systems were commonly identified, see eg 45,52,53,58,69 particularly to achieve an appropriate balance between redress for patients who suffer harm but also protecting practitioners from non-meritorious claims. ${ }^{36,49}$ Examples of reforms included caps on damages, ${ }^{11,50}$ specialist tribunals for medical claims/complaints ${ }^{46,66,70}$ and alternative compensation schemes. ${ }^{27,50,64}$ The development and promotion of evidence-informed practice guidelines was also recommended to avoid low value defensive practices..$^{40,48,50,54,59,71}$ System reforms to the culture of medicine were highlighted to shift away from over-treatment and could be complemented with public campaigns, such as Choosing Wisely initiatives, that challenge unnecessary tests, treatments and procedures. ${ }^{28,57,64}$ 
At organisational levels, proposed solutions focused on improving processes for handling complaints and legal claims, especially to improve efficiency, transparency, support for practitioners from management and peers, and early dispute resolution. ${ }^{15,30,65,72,73}$ Institutional culture change was also highlighted, including the need for role models and 'change champions' to challenge cultures of defensive practice and also to promote a nonpunitive quality improvement culture. ${ }^{11,17,18,32,34,39,68}$ Clear protocols and decision support systems for ordering tests and procedures were recommended. ${ }^{37-39}$ Working conditions that enable practitioners to avoid habitual patterns of defensive practice were also identified, including manageable workloads, adequate time for patient consultations, improved teamwork and continuity of care. ${ }^{29,42,55,74}$

At the micro level of individual practitioners, education and skills training were recommended, especially to support effective communication and shared decision-making with patients. ${ }^{32,63,66,71,72}$ Education should also reinforce that avoiding defensive practice is an aspect of medical professionalism and ensure practitioners understand their legal and ethical responsibilities, such as consent processes that involve adequately informing patients about treatment options, risks and benefits. ${ }^{48,51,67,75}$ Education could also provide practitioners with a realistic awareness of legal risks and counter exaggerated fears that drive defensive practice..$^{21,32,51,76,77}$

[insert Figure 4 here]

\section{DISCUSSION}

This review reveals a sizeable - and growing - body of empirical research on defensive medical practice. We note several limitations of this research, however promising trends and areas for potential policy and practice interventions can also be discerned to inform future research directions.

\section{Limitations of existing research}

First, many studies on defensive practice have been conducted in the United States and caution is needed in interpolating American data to other jurisdictions with differing cultures and systems of medicine and law. The body of American research may inaptly influence perceptions of legal risks in other countries. For instance, self-reported defensive 
practices among clinicians in other countries may reflect a cognitive availability bias framed around accounts of liability 'crises' from the United States. ${ }^{78}$

Second, the available knowledge on defensive practice is dominated by quantitative methods. Structured surveys are useful in providing descriptive data about defensive practices and their prevalence. Survey data may, however, underestimate the extent of defensive medicine that is practiced unconsciously and over-report defensive practices where doing so may advance respondents' interests in advocating for pro-doctor law and policy reforms. ${ }^{25}$ Response bias may occur where doctors with the most concerns about legal risks respond to the surveys. ${ }^{7}$ The framing effects of surveys can also distort results; how questions about defensive practice are framed has potentially as large an impact on self-reported defensive practice as does physician specialty and prior experience of malpractice litigation. ${ }^{79}$

Third, research on defensive practice has concentrated on a few medical fields, including surgery and obstetrics care. This focus is understandable given higher legal risks for practitioners in these fields. Defensive practice among general practitioners is comparatively under-explored, despite GPs' important gatekeeping role in ordering tests, making referrals, and prescribing.

\section{Trends}

A striking finding of our review is the marked increase in empirical research on defensive practice from 2011 onwards, especially in countries beyond the United States. Interestingly, this time period coincides with the launch of the Choosing Wisely initiative in the US and its spread to other countries, which has stimulated interest in connections between defensive practice and low value care. ${ }^{3,80}$ Choosing Wisely and similar campaigns, such as the British Medical Journal's Too Much Medicine program (http://www.bmj.com/too-much-medicine) and JAMA Internal Medicine's Less is More series (https://jamanetwork.com/collections/44045/less-is-more), bring increased attention to low value and unnecessary care and its multiple drivers, including fear of legal risks.

A majority of papers in this review framed defensive practice as driven by a fear of malpractice lawsuits, a reflection of the predominance of US research. How a problem is framed influences the conceptualisation of solutions ${ }^{81}$ and tort law reforms to deter 
lawsuits were commonly recommended. However, research on defensive practice increasingly considers a broader range of legal risks that may influence clinicians' behaviour, including patient complaints to healthcare organisations or professional regulatory agencies. This attention to risks beyond lawsuits is essential to understanding various sources of legal fear, which in turn illuminates targets for intervention at multiple levels, not just legislative reform. It is notable that defensive practice occurs even in jurisdictions, such as New Zealand and Belgium, where no-fault compensation schemes for medical injuries obviate negligence litigation. ${ }^{73,82}$ Moreover, tort law reforms appear to "have very little association with physicians' malpractice liability fears" ${ }^{\prime(p 1513)}$ and, as a consequence, the prevalence of defensive practice.

\section{Areas for future research}

The paucity of qualitative studies into defensive practice highlights a priority area for research, particularly to explore the internal, relational and environmental factors that influence clinicians' beliefs, attitudes and behaviours concerning legal risks. Purposive sampling could be beneficial to gain a more nuanced understanding of the characteristics and contexts of clinicians who are more or less likely to fear legal risks and engage in defensive practice. Measurement scales developed in previous studies ${ }^{60,71}$ could be used to identify practitioners who have heightened anxiety about legal risks, intolerance of uncertainty and tendency to defensive practice. Qualitative inquiry could reveal deeper insights into the practice behaviours of these clinicians and inform intervention research.

The reported pervasiveness of defensive medicine accentuates the need for evidence-based strategies to reduce and prevent the defensive provision of low- or no-value tests, treatments and referrals. Interventions targeting defensive behaviours are an important part of the broader de-implementation agenda in this area. ${ }^{83}$ Interventions should match clinician needs (eg, targeting highly defensive clinicians) as well as be tailored to the type of low value care that is being provided for defensive reasons. Verkerk and colleagues proposed a typology of low value interventions: ineffective care, or 'do not do' interventions that are not supported by evidence; inefficient care, that which is not clinically indicated for a particular patient; and unwanted care, that which is not in accordance with a patient's preferences. ${ }^{84}$ 
For the first category, an important message for clinicians is that providing 'do not do' interventions heightens legal risk. If a patient suffers harm from such interventions, the clinician's conduct cannot be defended as meeting a reasonable standard of care. ${ }^{85}$ Recent Australian research investigated hospital-acquired complications among patients unnecessarily admitted to hospital for low value procedures. ${ }^{86}$ This work highlights circumstances in which patients could seek legal redress for the harms of unnecessary care - and also notes the negative system impacts of misallocation of health services.

To support clinicians in reducing the defensive provision of inefficient care, multi-pronged strategies should focus on ensuring knowledge of current practice guidelines, reducing cognitive biases that drive low value interventions as defensive and habitual practices, ${ }^{78}$ and supporting appropriate monitoring and safety netting for patients who do not (yet) meet criteria for interventions. In the last category of unwanted care, strategies to support effective communication and shared decision-making will be needed in order to elicit and respect patient preferences.

Future studies can also elucidate the perceived sources of legal risks, especially in fields where practitioners worry about legal claims not just directly from their patients. For example, psychiatrists may fear legal responsibility if a patient experiencing mental illness harms a third party. Their defensive practices may involve judgements about the client's mental capacity and imposing restrictions on their liberty. ${ }^{17,42,74}$ In the end-of-life care context, life-sustaining medical interventions may be administered defensively to avoid the prospect of legal actions by bereaved family members. ${ }^{77}$ Ambiguity about diffuse legal obligations may trigger particular kinds of defensive behaviours that warrant further investigation.

Finally, some studies suggest that clinicians have an inaccurate understanding of the law 7,77 and propose that interventions to improve knowledge of the law and legal risks would help to reduce defensive practices. Future research provides an opportunity to test the hypothesis that "legal defensiveness and knowledge of medical law are inversely related." $77(\mathrm{p} 18)$ For instance, clinicians may need reassurance that the law does not impose an unattainable standard of perfection and "systems should recognise that good clinical judgment can at times result in bad patient outcomes." 7(p1516) Understanding legally acceptable "miss rates" 87 may also assuage doctors' fears about missed or delayed 
diagnoses. Interventions that mitigate dissonance between perceptions about how the law operates and how it actually applies could reduce hostile attitudes, such as viewing patients chiefly as potential complainants, not as partners in a therapeutic relationship.

\section{Limitations of our study}

This review was limited to papers that reported primary data collection on hedging-type defensive practices among physicians. Because of the explorative nature of the review and the diversity of included studies, a mapping review was the most suitable analytical approach, but precluded a critical appraisal of the quality of the studies and risk of bias. Moreover, the study identified important gaps in the literature, in particular few qualitative studies, fewer studies conducted outside the US and an underrepresentation of a number of fields of medical practice. These factors limit the generalisability of our findings. Papers using secondary analysis were excluded, such as analysis of medical claims data to identify trends in defensive behaviour. Our analysis does not cover studies that investigated other ways in which medico-legal concerns impact on physicians and their practices. While our focus was on medical practitioners, another recent review considered defensive practice by non-medical health professionals. ${ }^{88}$

\section{CONCLUSION}

Hedging-type defensive behaviours are reported by physicians who practice within different health care and legal systems. Our systemic analysis reveals intervention points at various levels and the findings of defensive practice across various countries reinforces the point that modifying the macro level legal framework is not sufficient to reduce and prevent defensive practice. We have proposed several key areas for future research, especially to investigate solutions to reduce defensive practice and its influence as a driver of low value care. 


\section{References}

1. Korenstein D. Medical Overuse as a Physician Cognitive Error: Looking Under the HoodMedical Overuse as a Cognitive ErrorResearch. JAMA Internal Medicine. 2019;179(1):26-27. doi:10.1001/jamainternmed.2018.5136

2. Brownlee S, Chalkidou K, Doust J, et al. Evidence for overuse of medical services around the world. The Lancet; London. 2017;390(10090):156-168. doi:http://dx.doi.org.ezproxy.lib.uts.edu.au/10.1016/S0140-6736(16)32585-5

3. Pathirana T, Clark J, Moynihan R. Mapping the drivers of overdiagnosis to potential solutions. BMJ. 2017;358:j3879. doi:10.1136/bmj.j3879

4. Saini V, Garcia-Armesto S, Klemperer D, et al. Drivers of poor medical care. The Lancet. 2017;390(10090):178-190. doi:10.1016/\$0140-6736(16)30947-3

5. Bourne $T$, Wynants $L$, Peters $M$, et al. The impact of complaints procedures on the welfare, health and clinical practise of 7926 doctors in the UK: a cross-sectional survey. BMJ Open. 2015;5(1):e006687-e006687. doi:10.1136/bmjopen-2014-006687

6. Agarwal R, Gupta A, Gupta S. The impact of tort reform on defensive medicine, quality of care, and physician supply: A systematic review. Health Services Research. 2019;54(4):851-859. doi:10.1111/1475-6773.13157

7. Reschovsky JD, Saiontz-Martinez CB. Malpractice Claim Fears and the Costs of Treating Medicare Patients: A New Approach to Estimating the Costs of Defensive Medicine. Health Services Research. 2018;53(3):1498-1516. doi:10.1111/1475-6773.12660

8. Hasson $\mathrm{H}$, Nilsen $\mathrm{P}$, Augustsson $\mathrm{H}$, von Thiele Schwarz U. Empirical and conceptual investigation of de-implementation of low-value care from professional and health care system perspectives: a study protocol. Implementation Science. 2018;13(1):67. doi:10.1186/s13012018-0760-7

9. Bates S, Clapton J, Coren E. Systematic maps to support the evidence base in social care. Evidence \& Policy; Bristol. 2007;3(4):539-551. doi:http://dx.doi.org.ezproxy.lib.uts.edu.au/10.1332/174426407782516484

10. Tebano G, Dyar OJ, Beovic B, Béraud G, Thilly N, Pulcini C. Defensive medicine among antibiotic stewards: the international ESCMID AntibioLegalMap survey. J Antimicrob Chemother. 2018;73(7):1989-1996. doi:10.1093/jac/dky098

11. Yan SC, Hulou MM, Cote DJ, et al. International Defensive Medicine in Neurosurgery: Comparison of Canada, South Africa, and the United States. World Neurosurgery. 2016;95:5361. doi:10.1016/j.wneu.2016.07.069

12. Badri M, Abdelbaky A, Yan G-X, Kowey PR. The impact of medical malpractice litigation on cardiovascular practice in the US and China. International Journal of Cardiology. 2014;177(1):48-50. doi:10.1016/j.ijcard.2014.09.158

13. Brilla R, Evers S, Deutschländer A, Wartenberg KE. Are neurology residents in the United States being taught defensive medicine? Clin Neurol Neurosurg. 2006;108(4):374-377.

doi:10.1016/j.clineuro.2005.05.013 
14. Bourne $T$, Vanderhaegen J, Vranken R, et al. Doctors' experiences and their perception of the most stressful aspects of complaints processes in the UK: an analysis of qualitative survey data. BMJ Open. 2016;6(7):e011711. doi:10.1136/bmjopen-2016-011711

15. Bourne T, De Cock B, Wynants L, et al. Doctors' perception of support and the processes involved in complaints investigations and how these relate to welfare and defensive practice: a cross-sectional survey of the UK physicians. BMJ Open. 2017;7(11):e017856. doi:10.1136/bmjopen-2017-017856

16. Ortashi O, Virdee J, Hassan R, Mutrynowski T, Abu-Zidan F. The practice of defensive medicine among hospital doctors in the United Kingdom. BMC Med Ethics. 2013;14(1):42. doi:10.1186/1472-6939-14-42

17. Passmore K, Leung W-C. Defensive practice among psychiatrists: a questionnaire survey. Postgrad Med J. 2002;78(925):671-673. doi:10.1136/pmj.78.925.671

18. Summerton N. Positive and negative factors in defensive medicine: a questionnaire study of general practitioners. BMJ. 1995;310(6971):27-29. doi:10.1136/bmj.310.6971.27

19. Summerton N. Trends in negative defensive medicine within general practice. Br J Gen Pract. 2000;50(456):565-566.

20. Symon A. Litigation and defensive clinical practice: quantifying the problem. Midwifery. 2000;16(1):8-14. doi:10.1054/midw.1999.0181

21. Symon A. Litigation and changes in professional behaviour: a qualitative appraisal. Midwifery. 2000;16(1):15-21. doi:10.1054/midw.1999.0193

22. Cheng YW, Snowden JM, Handler SJ, Tager IB, Hubbard AE, Caughey AB. Litigation in obstetrics: does defensive medicine contribute to increases in cesarean delivery? The Journal of MaternalFetal \& Neonatal Medicine. 2014;27(16):1668-1675. doi:10.3109/14767058.2013.879115

23. Rodriguez RM, Anglin D, Hankin A, et al. A longitudinal study of emergency medicine residents' malpractice fear and defensive medicine. Acad Emerg Med. 2007;14(6):569-573. doi:10.1197/j.aem.2007.01.020

24. Glassman PA, Rolph JE, Petersen LP, Bradley MA, Kravitz RL. Physicians' personal malpractice experiences are not related to defensive clinical practices. J Health Polit Policy Law. 1996;21(2):219-241. doi:10.1215/03616878-21-2-219

25. Klingman D, Localio AR, Sugarman J, et al. Measuring Defensive Medicine Using Clinical Scenario Surveys. J Health Polit Policy Law. 1996;21(2):185-220. doi:10.1215/03616878-21-2185

26. Ramella S, Mandoliti G, Trodella L, D'Angelillo RM. The first survey on defensive medicine in radiation oncology. Radiol Med. 2015;120(5):421-429. doi:10.1007/s11547-014-0465-1

27. Studdert DM, Mello MM, Sage WM, et al. Defensive medicine among high-risk specialist physicians in a volatile malpractice environment. JAMA. 2005;293(21):2609-2617. doi:10.1001/jama.293.21.2609

28. Hvidt EA, Lykkegaard J, Pedersen LB, Pedersen KM, Munck A, Andersen MK. How is defensive medicine understood and experienced in a primary care setting? A qualitative focus group 
study among Danish general practitioners. BMJ Open. 2017;7(12):e019851.

doi:10.1136/bmjopen-2017-019851

29. Brown SR, Brown J. Why Do Physicians Order Unnecessary Preoperative Tests? A Qualitative Study. Family Medicine. 2011;43(5):338-343.

30. Cunningham $W$, Dovey $S$. The effect on medical practice of disciplinary complaints: potentially negative for patient care. $N$ Z Med J. 2000;113(1121):464-467.

31. Cunningham W, Dovey S. Defensive changes in medical practice and the complaints process: a qualitative study of New Zealand doctors. N Z Med J. 2006;119(1244):U2283.

32. O'Leary KJ, Choi J, Watson K, Williams MV. Medical students' and residents' clinical and educational experiences with defensive medicine. Acad Med. 2012;87(2):142-148. doi:10.1097/ACM.0b013e31823f2c86

33. Weisman CS, Morlock LL, Teitelbaum MA, Klassen AC, Celentano DD. Practice changes in response to the malpractice litigation climate. Results of a Maryland physician survey. Med Care. 1989;27(1):16-24. doi:10.1097/00005650-198901000-00002

34. Chau T, Loertscher L. A multimodal high-value curriculum affects drivers of utilization and performance on the high-value care component of the internal medicine in-training exam. $J$ Community Hosp Intern Med Perspect. 2018;8(1):23-25. doi:10.1080/20009666.2018.1424488

35. Rothberg MB, Class J, Bishop TF, Friderici J, Kleppel R, Lindenauer PK. The cost of defensive medicine on 3 hospital medicine services. JAMA Intern Med. 2014;174(11):1867-1868. doi:10.1001/jamainternmed.2014.4649

36. Miller RA, Sampson NR, Flynn JM. The prevalence of defensive orthopaedic imaging: a prospective practice audit in Pennsylvania. J Bone Joint Surg Am. 2012;94(3):e18. doi:10.2106/JBJS.K.00646

37. Chen J, Majercik S, Bledsoe J, et al. The prevalence and impact of defensive medicine in the radiographic workup of the trauma patient: a pilot study. Am J Surg. 2015;210(3):462-467. doi:10.1016/j.amjsurg.2015.03.016

38. Rohacek M, Buatsi J, Szucs-Farkas Z, et al. Ordering CT pulmonary angiography to exclude pulmonary embolism: defense versus evidence in the emergency room. Intensive Care Med. 2012;38(8):1345-1351. doi:10.1007/s00134-012-2595-z

39. Katz DA, Williams GC, Brown RL, et al. Emergency Physicians' Fear of Malpractice in Evaluating Patients With Possible Acute Cardiac Ischemia. 2005;46:525-533.

40. Brateanu A, Schramm S, Hu B, et al. Quantifying the defensive medicine contribution to primary care costs. Journal of Medical Economics. 2014;17(11):810-816. doi:10.3111/13696998.2014.959125

41. Boven KV, Dijksterhuis $\mathrm{P}$, Lamberts $\mathrm{H}$. Defensive testing in Dutch family practice. Is the grass greener on the other side of the ocean? Journal of Family Practice. Published May 1, 1997. Accessed November 18, 2019. http://link.galegroup.com/apps/doc/A19454674/AONE?sid=Ims

42. Mullen R, Admiraal A, Trevena J. Defensive practice in mental health. NZ Med J. 2008;121(1286):85-91. 
43. Smith TR, Hulou MM, Yan SC, et al. Defensive medicine in neurosurgery: the Canadian experience. J Neurosurg. 2016;124(5):1524-1530. doi:10.3171/2015.6.JNS15764

44. Smith TR, Habib A, Rosenow JM, et al. Defensive Medicine in NeurosurgeryDoes State-Level Liability Risk Matter? Neurosurgery. 2015;76(2):105-114. doi:10.1227/NEU.0000000000000576

45. Roytowski D, Smith TR, Fieggen AG, Taylor A. Impressions of defensive medical practice and medical litigation among South African neurosurgeons. S Afr Med J. 2014;104(11):736-738.

46. Solaroglu I, Izci Y, Yeter HG, Metin MM, Keles GE. Health transformation project and defensive medicine practice among neurosurgeons in Turkey. PLOS ONE. 2014;9(10):e111446.

doi:10.1371/journal.pone.0111446

47. Nahed BV, Babu MA, Smith TR, Heary RF. Malpractice Liability and Defensive Medicine: A National Survey of Neurosurgeons. PLOS ONE. 2012;7(6):e39237.

doi:10.1371/journal.pone.0039237

48. Din R, Yan SC, Cote DJ, Acosta MA, Smith TR. Defensive Medicine in U.S. Spine Neurosurgery. Spine. 2017;42(3):177-185.

49. Cote DJ, Karhade AV, Larsen AMG, Castlen JP, Smith TR. Neurosurgical Defensive Medicine in Texas and Illinois: A Tale of 2 States. World Neurosurgery. 2016;89:112-120. doi:10.1016/j.wneu.2016.01.080

50. Asher E, Greenberg-Dotan S, Halevy J, Glick S, Reuveni H. Defensive Medicine in Israel - A Nationwide Survey. PLOS ONE. 2012;7(8):e42613. doi:10.1371/journal.pone.0042613

51. Osti M, Steyrer J. A national survey of defensive medicine among orthopaedic surgeons, trauma surgeons and radiologists in Austria: evaluation of prevalence and context. J Eval Clin Pract. 2015;21(2):278-284. doi:10.1111/jep.12305

52. Sathiyakumar V, Jahangir AA, Mir HR, et al. The prevalence and costs of defensive medicine among orthopaedic trauma surgeons: a national survey study. J Orthop Trauma.

2013;27(10):592-597. doi:10.1097/BOT.0b013e31828b7ab4

53. Sethi MK, Obremskey WT, Natividad H, Mir HR, Jahangir AA. Incidence and costs of defensive medicine among orthopedic surgeons in the United States: a national survey study. Am J Orthop. 2012;41(2):69-73.

54. Panella $\mathrm{M}$, Rinaldi $\mathrm{C}$, Leigheb $\mathrm{F}$, et al. Prevalence and costs of defensive medicine: a national survey of Italian physicians. J Health Serv Res Policy. 2017;22(4):211-217. doi:10.1177/1355819617707224

55. Tanriverdi O, Cay-Senler F, Yavuzsen T, et al. Perspectives and practical applications of medical oncologists on defensive medicine (SYSIPHUS study): a study of the Palliative Care Working Committee of the Turkish Oncology Group (TOG). Med Oncol. 2015;32(4):106. doi:10.1007/s12032-015-0555-5

56. Titus L, Reisch LM, Tosteson ANA, et al. Malpractice Concerns, Defensive Medicine, and the Histopathology Diagnosis of Melanocytic Skin Lesions. Am J Clin Pathol. 2018;150(4):338-345. doi:10.1093/ajcp/aqy057 
57. Reisch LM, Carney PA, Oster NV, et al. Medical malpractice concerns and defensive medicine: a nationwide survey of breast pathologists. Am J Clin Pathol. 2015;144(6):916-922.

doi:10.1309/AJCP80LYIMOOUJIF

58. Elli L, Tenca A, Soncini M, Spinzi G, Buscarini E, Conte D. Defensive medicine practices among gastroenterologists in Lombardy: between lawsuits and the economic crisis. Dig Liver Dis. 2013;45(6):469-473. doi:10.1016/j.dld.2013.01.004

59. Hiyama $\mathrm{T}$, Yoshihara $\mathrm{M}$, Tanaka $\mathrm{S}$, et al. Defensive medicine practices among gastroenterologists in Japan. World J Gastroenterol. 2006;12(47):7671-7675. doi:10.3748/wjg.v12.i47.7671

60. Benbassat J, Pilpel D, Schor R. Physicians' Attitudes Toward Litigation and Defensive Practice: Development of a Scale. Behavioral Medicine. 2001;27(2):52-60.

doi:10.1080/08964280109595771

61. Motta S, Testa D, Cesari U, Quaremba G, Motta G. Medical liability, defensive medicine and professional insurance in otolaryngology. BMC Res Notes. 2015;8. doi:10.1186/s13104-015$1318-2$

62. Johnston W, Rodriguez R, Suarez D, Fortman J. Study of Medical Students' Malpractice Fear and Defensive Medicine: A “Hidden Curriculum?" WestJEM. 2014;15(3):293-298.

doi:10.5811/westjem.2013.8.19045

63. Renkema E, Ahaus K, Broekhuis M, Tims M. Triggers of defensive medical behaviours: a crosssectional study among physicians in the Netherlands. BMJ Open. 2019;9(6).

doi:10.1136/bmjopen-2018-025108

64. Reuveni I, Pelov I, Reuveni H, Bonne O, Canetti L. Cross-sectional survey on defensive practices and defensive behaviours among Israeli psychiatrists. BMJ Open. 2017;7(3):e014153. doi:10.1136/bmjopen-2016-014153

65. Nakamura N, Yamashita Y. Malpractice lawsuits and change in work in Japanese surgeons. J Surg Res. 2015;193(1):210-216. doi:10.1016/j.jss.2014.08.029

66. Saint S, Vaughn VM, Chopra V, Fowler KE, Kachalia A. Perception of Resources Spent on Defensive Medicine and History of Being Sued Among Hospitalists: Results from a National Survey. J Hosp Med. 2018;13(1):26-29. doi:10.12788/jhm.2800

67. Zhu L, Li L, Lang J. The attitudes towards defensive medicine among physicians of obstetrics and gynaecology in China: a questionnaire survey in a national congress. BMJ Open. 2018;8(2). doi:10.1136/bmjopen-2017-019752

68. Catino M. Blame culture and defensive medicine. Cogn Tech Work. 2009;11(4):245. doi:10.1007/s10111-009-0130-y

69. Bishop TF, Federman AD, Keyhani S. Physicians' Views on Defensive Medicine: A National Survey. Arch Intern Med. 2010;170(12):1081-1083. doi:10.1001/archinternmed.2010.155

70. Küçük M. Defensive medicine among obstetricians and gynaecologists in Turkey. Journal of Obstetrics and Gynaecology. 2018;38(2):200-205. doi:10.1080/01443615.2017.1340933 
71. Brodsky SL, Cramer RJ. Concerns about litigation: Conceptualization, development, and measurement. Journal of Psychiatry and Law. 2008;36(4):525-542.

72. Lee M. The Effects of Criminal Punishment on Medical Practices in the Medical Environment. Int J Environ Res Public Health. 2019;16(4). doi:10.3390/ijerph16040604

73. Vandersteegen T, Marneffe W, Cleemput I, Vandijck D, Vereeck L. The determinants of defensive medicine practices in Belgium. Health Economics, Policy and Law. 2017;12(3):363386. doi:10.1017/S174413311600030X

74. Krawitz R, Batcheler M. Borderline Personality Disorder: A Pilot Survey about Clinician Views on Defensive Practice. Australasian Psychiatry. 2006;14(3):320-322.

75. Silberstein E, Shir-Az O, Reuveni H, et al. Defensive Medicine Among Plastic and Aesthetic Surgeons in Israel. Aesthet Surg J. 2016;36(10):NP299-NP304. doi:10.1093/asj/sjw094

76. Salem O, Forster C. Defensive medicine in general practice: recent trends and the impact of the Civil Liability Act 2002 (NSW). J Law Med. 2009;17(2):235-248.

77. McCrary SV, Swanson JW. Physicians' legal defensiveness and knowledge of medical law: comparing Denmark and the USA. Scand J Public Health. 1999;27(1):18-21.

78. Scott IA, Soon J, Elshaug AG, Lindner R. Countering cognitive biases in minimising low value care. The Medical Journal of Australia. 2017;206(9):407-411. doi:10.5694/mja16.00999

79. Baicker K, Wright BJ, Olson NA. Reevaluating Reports of Defensive Medicine. Journal Of Health Politics, Policy And Law. 2015;40(6):1157-1177. doi:10.1215/03616878-3424462

80. Ries NM. Choosing Wisely: Law's Contribution as a Cause of and a Cure for Unwise Health Care Choices. Journal of Law and Medicine. 2017;25(1):210-228.

81. Koon AD, Hawkins B, Mayhew SH. Framing and the health policy process: a scoping review. Health Policy Plan. 2016;31(6):801-816. doi:10.1093/heapol/czv128

82. Wallis KA. No-fault, no difference: no-fault compensation for medical injury and healthcare ethics and practice. Br J Gen Pract. 2017;67(654):38-39. doi:10.3399/bjgp17X688777

83. Parker G, Rappon T, Berta W. Active change interventions to de-implement low-value healthcare practices: a scoping review protocol. BMJ Open. 2019;9(3):e027370. doi:10.1136/bmjopen-2018-027370

84. Verkerk EW, Tanke MAC, Kool RB, van Dulmen SA, Westert GP. Limit, lean or listen? A typology of low-value care that gives direction in de-implementation. Int I Qual Health Care. 2018;30(9):736-739. doi:10.1093/intqhc/mzy100

85. Bester JC. Defensive practice is indefensible: how defensive medicine runs counter to the ethical and professional obligations of clinicians. Med Health Care and Philos. 2020;23(3):413420. doi:10.1007/s11019-020-09950-7

86. Badgery-Parker T, Pearson S-A, Dunn S, Elshaug AG. Measuring Hospital-Acquired Complications Associated With Low-Value Care. JAMA Intern Med. 2019;179(4):499-505. doi:10.1001/jamainternmed.2018.7464 
87. Brooker JA, Hastings JW, Major-Monfried H, et al. The Association Between Medicolegal and Professional Concerns and Chest Pain Admission Rates. Academic Emergency Medicine.

2015;22(7):883-886. doi:10.1111/acem.12708

88. Rinaldi C, D'Alleva A, Leigheb F, et al. Defensive practices among non-medical health professionals: An overview of the scientific literature. Journal of Healthcare Quality Research. 2019;34(2):97-108. doi:10.1016/j.jhqr.2018.12.005 
Figures and Tables

Figure 1: Literature search flow diagram

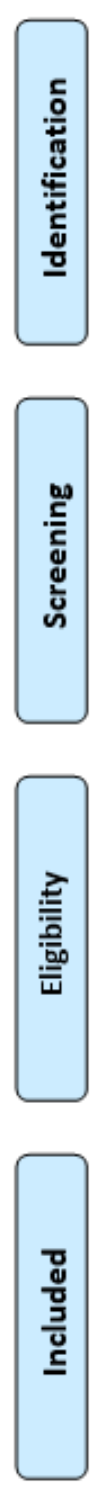
2166 records identified through database searching

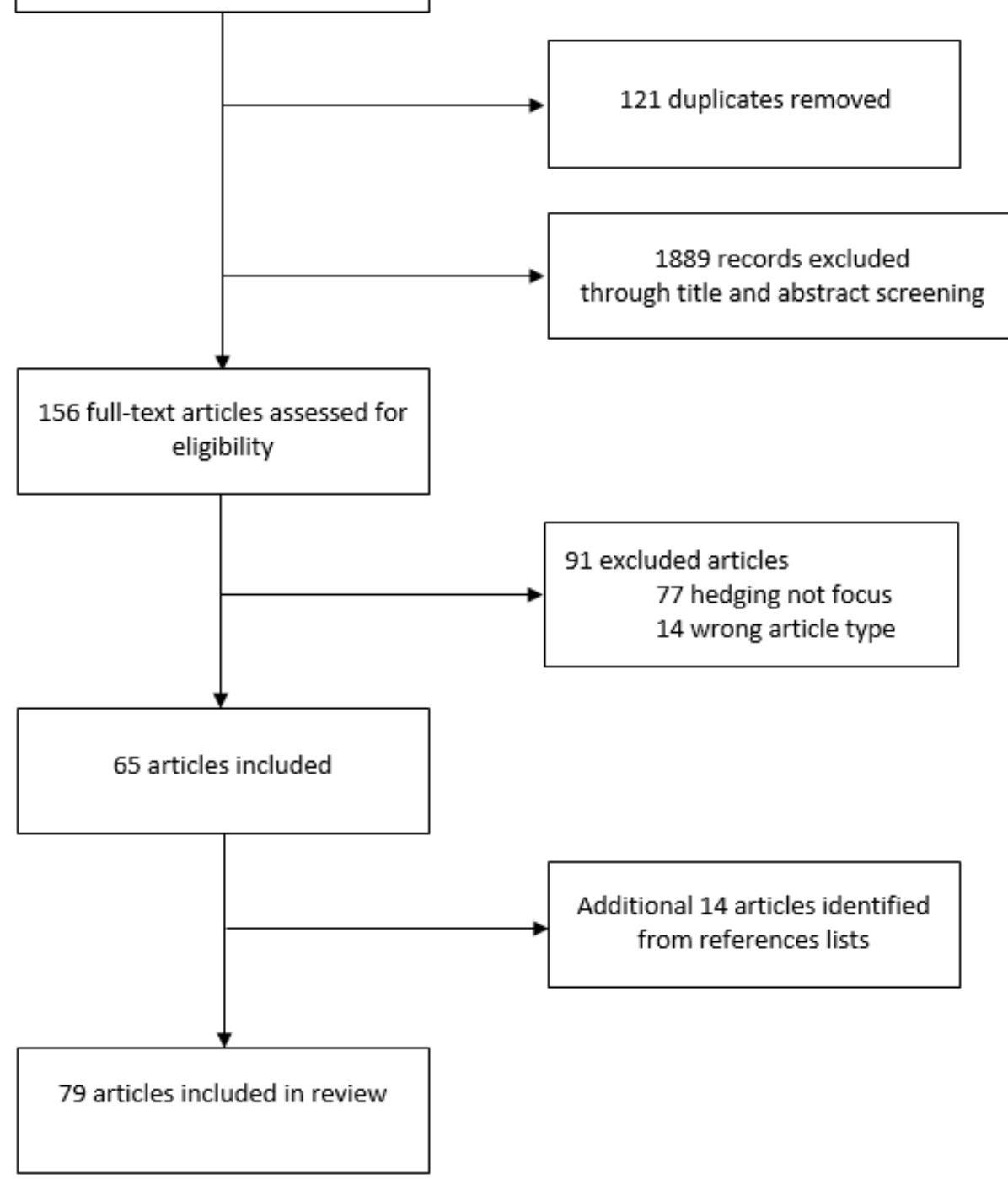


Figure 2: Country of Study

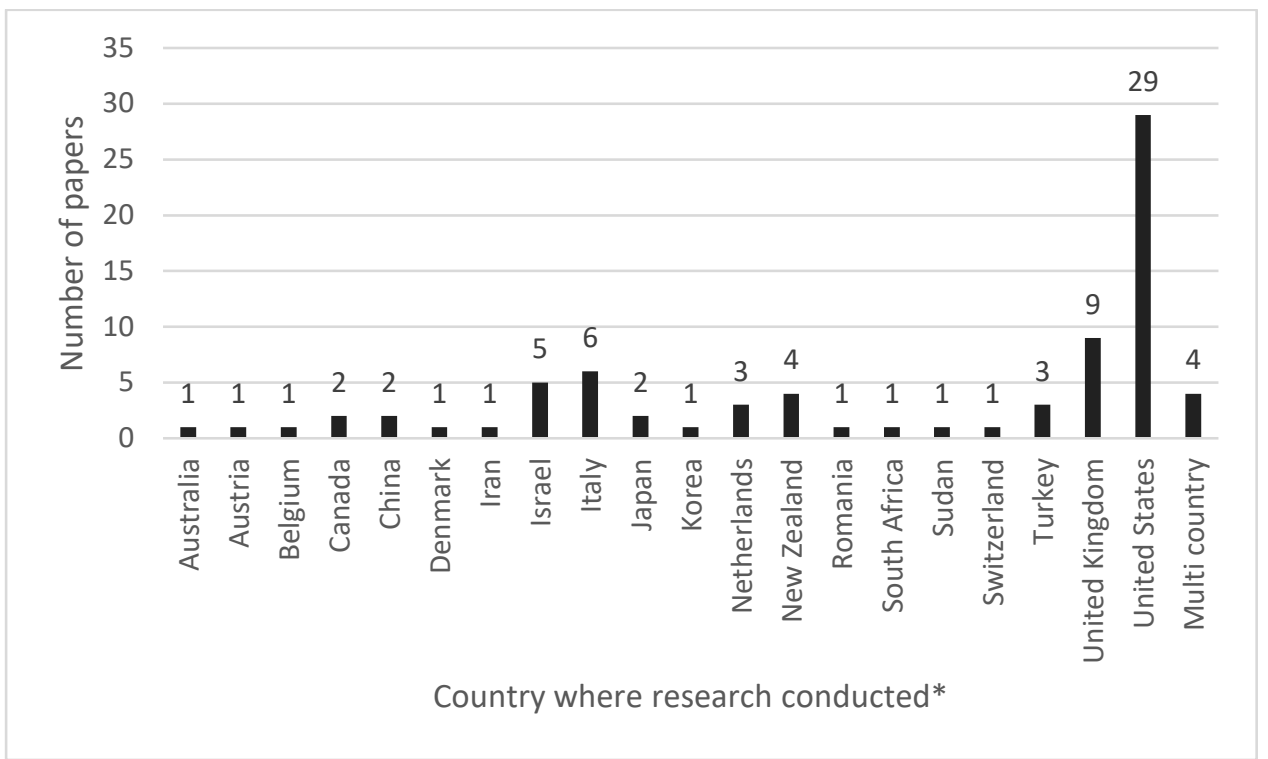

*Studies involving data collection in a single country; multi-country studies counted separately. 
Figure 3: Year of Publication

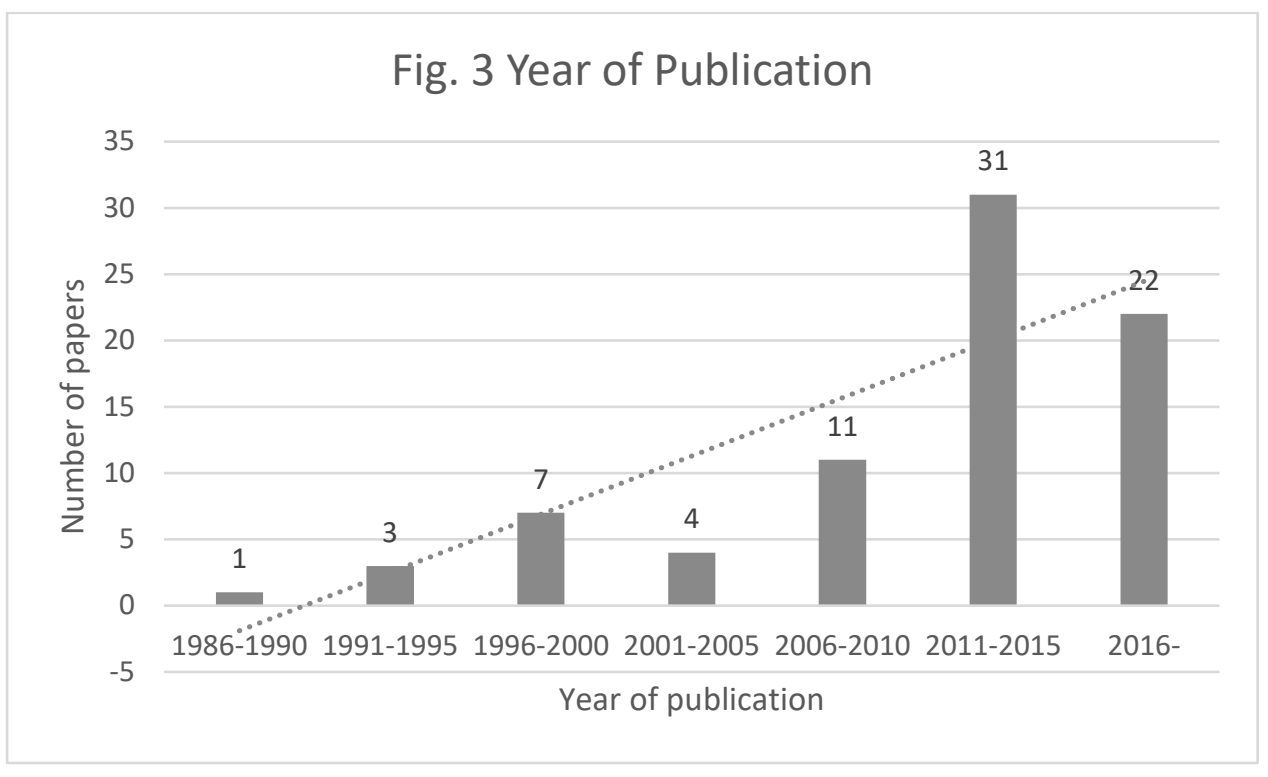


Table 1. Fields of practice covered in $>5$ papers

\begin{tabular}{|l|c|c|}
\hline \multicolumn{2}{|c|}{ Participants in Studies } \\
\hline Mixed sample* & \multicolumn{2}{|c|}{21 papers } \\
\hline Field of practice & Single sample $^{*}$ & 15 \\
\hline Surgery - all fields & 16 & 3 \\
\hline Neurosurgery & 9 & 9 \\
\hline General surgery /specialty not indicated & 2 & 2 \\
\hline Trauma and/or orthopaedic surgery & 4 & 6 \\
\hline Plastic / aesthetic surgery & 1 & 4 \\
\hline $\begin{array}{l}\text { Obstetric care (included studies of ob-gyns, GPs who deliver } \\
\text { babies, midwives) }\end{array}$ & 8 & 1 \\
\hline General / family practice, primary care & 9 & 1 \\
\hline Emergency medicine & 4 & \\
\hline Psychiatry / mental health & 4 & \\
\hline
\end{tabular}

* Mixed sample refers to studies where respondents were recruited from multiple fields of medical practice. Single sample refers to studies where respondents were from one field of practice. 
Figure 4. Potential Solutions to Reduce Defensive Practice

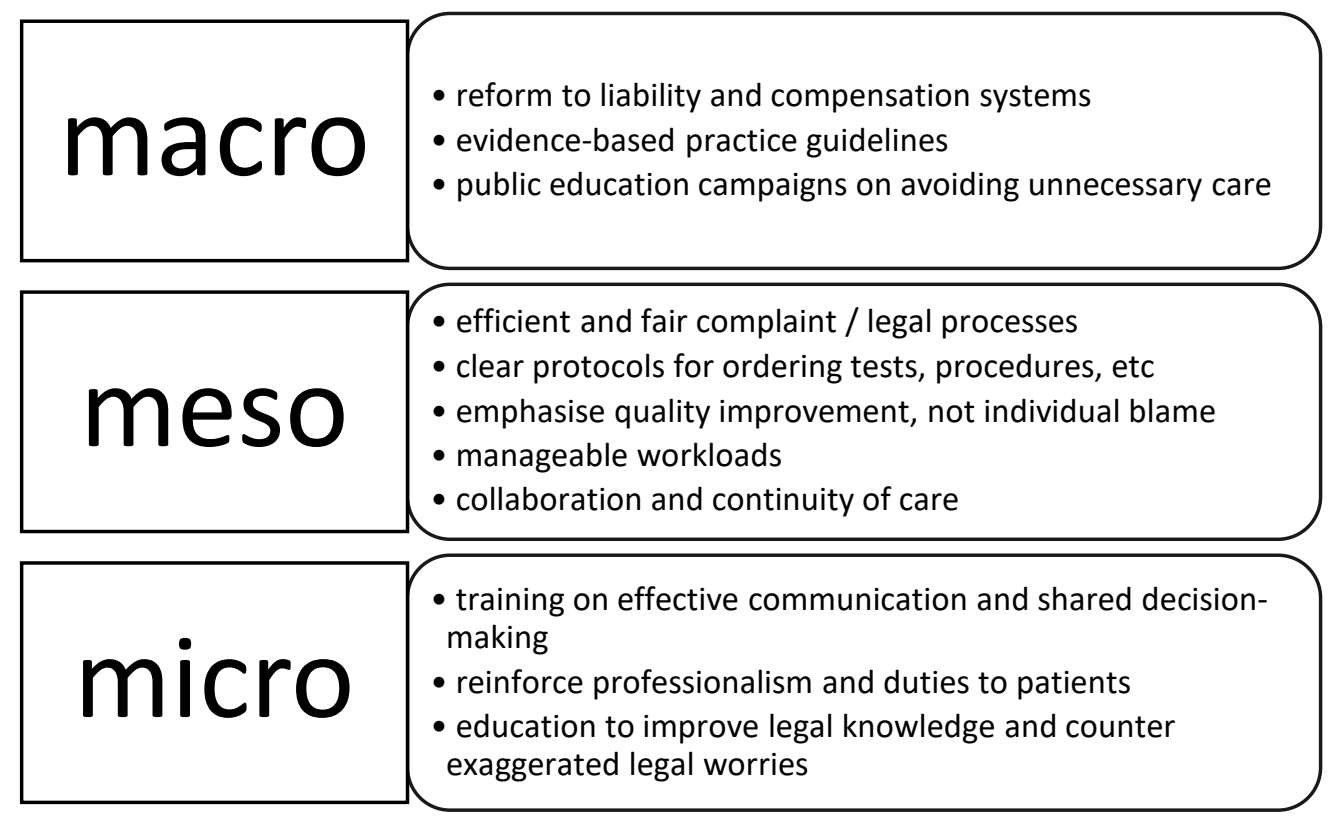

